TRANSACTIONS OF THE

AMERICAN MATHEMATICAL SOCIETY

Volume 357, Number 11, Pages 4249-4268

S 0002-9947(05)04031-6

Article electronically published on June 13, 2005

\title{
HAUSDORFF MEASURES, DIMENSIONS AND MUTUAL SINGULARITY
}

\author{
MANAV DAS
}

\begin{abstract}
Let $(X, d)$ be a metric space. For a probability measure $\mu$ on a subset $E$ of $X$ and a Vitali cover $V$ of $E$, we introduce the notion of a $b_{\mu^{-}}$ Vitali subcover $V_{\mu}$, and compare the Hausdorff measures of $E$ with respect to these two collections. As an application, we consider graph directed selfsimilar measures $\mu$ and $\nu$ in $\mathbb{R}^{d}$ satisfying the open set condition. Using the notion of pointwise local dimension of $\mu$ with respect to $\nu$, we show how the Hausdorff dimension of some general multifractal sets may be computed using an appropriate stochastic process. As another application, we show that Olsen's multifractal Hausdorff measures are mutually singular.
\end{abstract}

\section{INTRODUCTION}

Let $(X, d)$ be a metric space. Let $F \subseteq X, \alpha>0$. The $\alpha$-dimensional Hausdorff measure is defined as

$\mathcal{H}^{\alpha}(F)=\sup _{\epsilon>0} \inf \left\{\sum_{j} \operatorname{diam}\left(I_{j}\right)^{\alpha}: F \subseteq \bigcup_{j} I_{j}, \operatorname{diam}\left(I_{j}\right)<\epsilon, I_{j}\right.$ is any set in $\left.X\right\}$.

The class of sets used to define the measure is much too large. Often we are interested in finding an appropriate subclass which would give us the same measure, or differ from the original measure by at most a constant factor. One way to obtain a subclass is to consider the centered Hausdorff measure, defined as follows:

$\widetilde{\mathcal{C}}^{\alpha}(F)=\sup _{\epsilon>0} \inf \left\{\sum_{j} \operatorname{diam}\left(B_{j}\left(x_{j}\right)\right)^{\alpha}: F \subseteq \bigcup_{j} B_{j}\left(x_{j}\right), x_{j} \in F, \operatorname{diam}\left(B_{j}\left(x_{j}\right)\right)<\epsilon\right\}$,

where $B_{j}\left(x_{j}\right)$ is a closed ball in $X$ and

$$
\mathcal{C}^{\alpha}(F)=\sup _{E \subseteq F} \widetilde{\mathcal{C}}^{\alpha}(E) .
$$

It turns out that the centred Hausdorff measure is within a constant multiple of the ordinary Hausdorff measure, and so the two dimensions coincide. We consider a more general Hausdorff measure as follows:

Let $V$ be a Vitali cover for $F \subseteq X$, and let $\mathcal{P}(X)$ denote the family of Borel probability measures on $X$. For $\mu \in \mathcal{P}(X)$, we define the $\mu$-Hausdorff measure

Received by the editors May 19, 1997.

2000 Mathematics Subject Classification. Primary 28A78; Secondary 28A80, 60A10.

Key words and phrases. Hausdorff measure, Vitali cover, multifractal, (strong) open set condition, stochastic process, stoppings.

(C)2005 American Mathematical Society Reverts to public domain 28 years from publication 
(with respect to $V$ ) as follows:

$$
\mathcal{H}_{\mu}^{\alpha}(F: V)=\sup _{\epsilon>0} \inf \left\{\sum_{j} \mu\left(I_{j}\right)^{\alpha}: F \subseteq \bigcup_{j} I_{j}, \mu\left(I_{j}\right)<\epsilon, I_{j} \in V, I_{j} \cap F \neq \emptyset\right\} .
$$

Let $\operatorname{dim}_{\mu}(F: V)$ denote the corresponding Hausdorff dimension. When we take $V$ to be the collection of all closed balls centered in $F$, we will simply write $\mathcal{H}_{\mu}^{\alpha}(F)$ and $\operatorname{dim}_{\mu} F$. Given a Vitali cover $V$ of $\operatorname{supp} \mu$, we introduce the notion of a $b_{\mu}$-Vitali subcover $V_{\mu}$, and show that for any $E \subseteq \operatorname{supp} \mu$,

$$
\mathcal{H}_{\mu}^{\alpha}(E: V) \leq \mathcal{H}_{\mu}^{\alpha}\left(E: V_{\mu}\right) \leq \mathcal{H}_{\mu}^{\alpha b_{\mu}}(E: V)
$$

We intend to show the existence of a $b_{\mu}$-Vitali subcover in a special setting, and show two applications of the result obtained above. In order to motivate this, allow me to digress and quickly summarize another line of thought.

Fractal measures are most often constructed using an iterative process. Thus the symbol space, or the string space, is of paramount importance in the analysis of the measure. One of the consequences of the iterative process is the construction of a collection of sets $C$, called cylinders. Computations involving the measure are more naturally carried out by using these cylinder sets. But for a given metric structure, we are really interested in deducing the behaviour of the measure with respect to the closed balls, $B$. This immediately leads us to ask if the Hausdorff dimensions computed using these two classes are equal. Billingsley 2] proved this for certain subsets of $\mathbb{R}$. Cutler [4] introduced the notion of bounded Vitali covers, and extended Billingsley's idea to include a larger class of subsets of the real line.

Consider now the following problem: Let $\mu$ be a Borel probability measure on $X$ and let $B_{\epsilon}(x)$ be the closed ball of radius $\epsilon$ centered at $x$. For $x \in X$ define the local dimension of $\mu$ at $x$ as

$$
\alpha_{\mu}(x)=\lim _{\epsilon \rightarrow 0} \frac{\log \mu B_{\epsilon}(x)}{\log \operatorname{diamB}_{\epsilon}(\mathrm{x})},
$$

if the limit exists. For $\alpha \geq 0$, consider the set

$$
K^{(\alpha)}=\left\{x \in \operatorname{supp} \mu: \alpha_{\mu}(\mathrm{x})=\alpha\right\},
$$

where supp $\mu$ is taken to be the topological support of $\mu$. Typically, the sets $K^{(\alpha)}$ are dense in supp $\mu$. Intuitively, for fixed $\alpha$, we may think of $K^{(\alpha)}$ as the collection of points where the measure scales as $\alpha$, i.e. $\mu B_{\epsilon}(x) \asymp \epsilon^{\alpha}$. What can be said about the Hausdorff dimension of $K^{(\alpha)}$ ? Although not much is known for general metric spaces and arbitrary measures, much has been accomplished from the point of view of multifractal theory. Halsey et al. 12] argued that certain fractals carrying a natural measure may be analyzed in terms of the scaling properties of the measure, i.e. they may be decomposed into multifractals. Cawley and Mauldin [3] were the first to provide a geometric measure-theoretical framework for such a decomposition for Moran fractals. Edgar and Mauldin 10] carried out the analysis for graph directed self-similar measures in $\mathbb{R}^{d}$ with totally disconnected support. Olsen [16] proposed a multifractal formalism that included the above analyses. Olsen's paper also contains a comprehensive account of the development of the theory and lists some important references. Attention has primarily focussed on the situation where $X$ is taken to be a $d$-dimensional Euclidean space, and $\mu$ is a self-similar measure with totally disconnected support. Arbeiter and Patzschke [1] considered random 
self-similar sets satisfying the open set condition. In much of the literature, the Hausdorff measure is calculated either by using the mass distribution principle or by explicit estimates. In this paper, we show how Billingsley's idea may be used to compute the Hausdorff dimension of $K^{(\alpha)}$, where $\mu$ is taken to be a graphdirected self-similar measure in $\mathbb{R}^{d}$ satisfying the open set condition, thus answering questions posed in [10 Question (d)], [16 Question 7.8], and [15, Page 58], among others. We are aided in our analysis by explicit relations between closed balls and cylinders, as obtained in [7].

Let us now return to the original idea of a $b_{\mu}$-Vitali subcover. As an application, we consider a more general local dimension. Let $\mu, \nu$ be two graph-directed selfsimilar measures satisfying the open set condition. For $\alpha>0$, we are interested in the Hausdorff dimension of the following set:

$$
K^{(\alpha)}=\left\{x \in \operatorname{supp} \mu: \lim _{\epsilon \rightarrow 0} \frac{\log \mu\left(B_{\epsilon}(x)\right)}{\log \nu\left(B_{\epsilon}(x)\right)}=\alpha\right\} .
$$

It turns out that in order to compare the collections $B$ and $C$ in this case, we need to first compare subcollections $B_{\mu}$ and $C_{\mu}$, which incidentally turn out to be $b_{\mu}$-Vitali subcovers.

As another application, we consider Olsen's multifractal Hausdorff measures, generalize them and give an answer to his question [16, Question 7.9] in this more general setting.

\section{2. $b_{\mu}$-Vitali COVER}

We introduce the notion of a $b_{\mu}$-Vitali subcover of a Vitali cover $V$.

Definition 2.1. Let $\mu$ be any probability measure on $X$, let $F \subseteq \operatorname{supp} \mu$, let $V$ be any Vitali cover for $F$, and let $V_{\mu}$ be a subcollection of $V$ such that:

For each $x \in F$, there exists a nested sequence of sets from $V$ denoted $\left(I_{j}(x)\right)_{j}$ such that

(1) $x \in I_{j}(x)$ for each $j$.

(2) $\mu\left(I_{j}(x)\right) \rightarrow 0$ as $j \rightarrow \infty$.

(3) For any $x \in F$ and any $B \in V$ with $x \in B$, there exists $j \in \mathbb{N}$ ( $j$ dependent on $x)$ with $I_{j+1}(x) \subseteq B \subseteq I_{j}(x)$.

(4) $\inf _{j}\left(\log \mu\left(I_{j}(x)\right) / \log \mu\left(I_{j+1}(x)\right)\right) \geq b_{\mu}, 0<b_{\mu} \leq 1$.

Let $V_{\mu}=\left(I_{j}(x)\right)_{j \in \mathbb{N}, x \in F}$. If $V_{\mu}$ exists, then we will call it a $b_{\mu}$-Vitali covering of $F$ with respect to $V$.

Theorem 2.2. Let $V$ be a Vitali cover for $F=\operatorname{supp} \mu$, and suppose there is a subcollection $V_{\mu}$ which is a $b_{\mu}$-Vitali subcover of $F$ with respect to $V$. Then for any $E \subseteq F$

$$
\mathcal{H}_{\mu}^{\alpha}(E: V) \leq \mathcal{H}_{\mu}^{\alpha}\left(E: V_{\mu}\right) \leq \mathcal{H}_{\mu}^{\alpha b_{\mu}}(E: V)
$$

Proof. Clearly,

$$
\mathcal{H}_{\mu}^{\alpha}(E: V) \leq \mathcal{H}_{\mu}^{\alpha}\left(E: V_{\mu}\right)
$$

since $V_{\mu}$ is a subclass of $V$. For the reverse inequality, consider the set

$$
E_{k, m, n}=\left\{x \in E: \mu\left(I_{m}(x)\right)>\frac{1}{n} \text { and } j \geq m \Rightarrow \frac{\log \mu\left(I_{j}(x)\right)}{\log \mu\left(I_{j+1}(x)\right)} \geq b_{\mu}-\frac{1}{k}\right\}
$$


Then,

$$
E=\bigcup_{k} \bigcup_{m} E_{k, m, n}
$$

Fix $k, m, n \in \mathbb{N}$. Let $\rho \leq \frac{1}{n}$ and let $\left(B_{i}\right)$ be a $\mu-\rho$ cover of $E_{k, m, n}$ using sets from $V$. By the definition of a $b_{\mu}$-Vitali subcover, we can choose $j(i)$ such that $I_{j(i)+1}\left(x_{i}\right) \subseteq B_{i} \subseteq I_{j(i)}\left(x_{i}\right)$. By our choice of $\rho$, we must have $j(i) \geq m$. Since $x_{i} \in E_{k, m, n}$, we get

$$
\begin{aligned}
\frac{\log \mu\left(I_{j(i)}\left(x_{i}\right)\right)}{\log \mu\left(I_{j(i)+1}\left(x_{i}\right)\right)} & \geq b_{\mu}-\frac{1}{k} \\
\Rightarrow \sum \mu\left(I_{j(i)}\left(x_{i}\right)\right)^{\alpha} & \leq \sum \mu\left(I_{j(i)+1}\left(x_{i}\right)\right)^{\alpha\left(b_{\mu}-\frac{1}{k}\right)} \leq \sum \mu\left(B_{i}\right)^{\alpha\left(b_{\mu}-\frac{1}{k}\right)} \\
\Rightarrow \mathcal{H}_{\mu}^{\alpha}\left(E_{k, m, n}: V_{\mu}\right) & \leq \mathcal{H}_{\mu}^{\alpha\left(b_{\mu}-\frac{1}{k}\right)}\left(E_{k, m, n}: V\right) \\
\Rightarrow \mathcal{H}_{\mu}^{\alpha}\left(E: V_{\mu}\right) & \leq \mathcal{H}_{\mu}^{\alpha b_{\mu}}(E: V) .
\end{aligned}
$$

Corollary 2.3. Let $V$ be a Vitali cover for $F=\operatorname{supp} \mu$, and let $V_{\mu}$ be any corresponding $b_{\mu}$-Vitali subcover of $F$ with respect to $V$. Then for any $E \subseteq F$,

$$
b_{\mu} \operatorname{dim}_{\mu}\left(E: V_{\mu}\right) \leq \operatorname{dim}_{\mu}(E: V) \leq \operatorname{dim}_{\mu}\left(E: V_{\mu}\right) .
$$

We may strengthen condition 4 in Definition 2.1 to require that:

$\left(4^{\prime}\right) \lim _{j} \frac{\log \mu\left(I_{j}(x)\right)}{\log \mu\left(I_{j+1}(x)\right)}=b_{\mu}$.

Let $V_{\mu}=\left(I_{j}(x)\right)_{j \in \mathbb{N}, x \in F}$. Then we will call $V_{\mu}$ a strong $b_{\mu}$-Vitali covering of $F$ with respect to $V$.

We may now obtain stronger conclusions in Theorem 2.2 and Corollary 2.3. We state them without the proofs:

Theorem 2.4. Let $V$ be a Vitali cover for $F=\operatorname{supp} \mu$, and let $V_{\mu}$ be any corresponding strong $b_{\mu}$-Vitali cover of $F$ with respect to $V$. Then for any $E \subseteq F$,

$$
\mathcal{H}_{\mu}^{\alpha}\left(E: V_{\mu}\right)=\mathcal{H}_{\mu}^{\alpha b_{\mu}}(E: V) .
$$

Corollary 2.5. Let $V$ be a Vitali cover for $F=\operatorname{supp} \mu$, and let $V_{\mu}$ be any corresponding strong $b_{\mu}$-Vitali cover of $F$ with respect to $V$. Then for any $E \subseteq F$,

$$
b_{\mu} \operatorname{dim}_{\mu}\left(E: V_{\mu}\right)=\operatorname{dim}_{\mu}(E: V) .
$$

\section{HAUSDORFF DIMENSION I}

In this section, we describe how the Hausdorff dimensions may be computed using an appropriate stochastic process. When we realize the stochastic process in the Euclidean space, the natural sets are called cylinder sets, as for instance, the $r$-adic intervals, or the line segments obtained in the construction of the classical Cantor set. Now the Hausdorff dimension computed using these cylinder sets may not be the same as the one computed using closed balls. We will show that the two dimensions actually coincide when we use graph directed self-similar measures. We first investigate the $\mu$-Hausdorff dimension of a set with respect to the Lebesgue measure. This will motivate the difficulties that arise when computing the $\mu$-Hausdorff measure with respect to another measure $\nu$. 
3.1. Preliminaries. Let $\left\{x_{1}, x_{2}, \ldots\right\}$ be a stochastic process defined on a probability measure space $(\Omega, \mathcal{B}, \mu)$. Suppose that the state space of the process is a finite set $\sigma$, the states of which are given by $\sigma=\{1,2, \ldots, N\}$. An $n$-cylinder is a set of the form

$$
\left\{\omega: x_{k}(\omega)=a_{k}, k=1,2, \ldots, n\right\},
$$

where $\left(a_{1}, a_{2}, \ldots, a_{n}\right)$ is a sequence of states. Let $C$ denote the collection of all such $n$-cylinders, where $n=1,2, \ldots$. The cylinder Hausdorff measure was introduced by Billingsley in [2]. Let $\operatorname{dim}_{\mu}(M: C)$ denote the corresponding cylinder Hausdorff dimension. Billingsley proved the following result.

Theorem 3.1. Let $\mu, \gamma$, be any two measures corresponding to the same stochastic process. If

then

$$
M \subseteq\left\{\omega \in \Omega: \lim _{n} \frac{\log \gamma\left(c_{n}(\omega)\right)}{\log \mu\left(c_{n}(\omega)\right)}=\theta\right\},
$$

$$
\operatorname{dim}_{\mu}(M: C)=\theta \operatorname{dim}_{\gamma}(M: C) .
$$

We now introduce the space of strings and the relevant sets and measures. Let $(V, E)$ be a directed multigraph, where $V$ is the set of vertices and $E$ is the set of edges. For $u, v \in V$, there is a subset $E_{u v}$ of $E$ known as the edges from $u$ to $v$. Let $E_{u}=\bigcup_{v \in V} E_{u v}$. For $e \in E$, let $i(e)$ denote the initial vertex of $e$, and $t(e)$ be the terminal vertex of $e$. A path in the graph is a finite string $\gamma=\gamma_{1} \gamma_{2} \ldots \gamma_{k}$ of edges, such that $t\left(\gamma_{i}\right)=i\left(\gamma_{i+1}\right)$. Let $E_{u v}^{(k)}$ be the set of all paths of length $k$ that begin at $u$ and end at $v$. Let $E_{u}^{(k)}$ be the set of all paths that are of length $k$ and begin at $u$. Let $E_{u}^{\star}$ be the set of all finite paths of any length that begin at $u$ and $E^{\star}=\bigcup_{u \in V} E_{u}^{\star}$. An infinite string over the alphabet $E$ corresponds to an infinite path if the terminal vertex for each edge matches the initial vertex of the next edge. Let $E_{u}^{(\omega)}$ be the set of all infinite paths starting at $u \in V$, and let $E^{(\omega)}=\bigcup_{u \in V} E_{u}^{(\omega)}$. For $\alpha \in E^{\star}, \sigma \in E^{(\omega)}$, we say that $\alpha \prec \sigma$, if there exists $\tau \in E_{t(\alpha)}^{(\omega)}$, such that $\sigma=\alpha \tau$. If $\alpha \in E^{\star}$, let

$$
[\alpha]=\left\{\sigma \in E^{(\omega)}: \alpha \prec \sigma\right\}
$$

and we call it the cylinder set generated by $\alpha$. Let $\sigma \mid k$ denote the finite string comprising of the first $k$ symbols of $\sigma$.

Let $G$ be a $M W$ Graph with probabilities (see [9]). $G$ is said to satisfy the open set condition $(O S C)$ iff there exists a list $\left(U_{u}\right)_{u \in V}$ of sets, where $U_{u}$ is a non-empty, open and bounded subset of $X_{u}$ satisfying

(1) $\bigcup_{e \in E_{u}} S_{e}\left(U_{t(e)}\right) \subset U_{u}, \forall u \in V$.

(2) $S_{e}\left(U_{t(e)}\right) \cap S_{e^{\prime}}\left(U_{t\left(e^{\prime}\right)}\right)=\emptyset, \forall u \in V, e, e^{\prime} \in E_{u}, e \neq e^{\prime}$.

Further, if $U_{u} \cap K_{u} \neq \emptyset, \forall u \in V$, then $G$ is said to satisfy the strong $O S C$ (SOSC).

By the boundary of a set $A \subseteq \mathbb{R}^{d}$, denoted $\partial A$, we will mean the collection of all points belonging to the closure of the set $A$ and to the closure of the complement of $A$ in $\mathbb{R}^{d}$. Let $J_{u}$ be a non-empty compact subset of $X_{u}$. Its existence has been guaranteed by Schief [19] and Wang [21]. Since $\operatorname{int}\left(J_{u}\right) \neq \emptyset, \mathcal{L}^{d}\left(J_{u}\right)>0$, where $\mathcal{L}^{d}$ denotes the $d$-dimensional Lebesgue measure. For $e=e_{1} e_{2} \ldots e_{n} \in E^{\star}$, we let $S(e)=S_{e_{1}} \circ S_{e_{2}} \circ \ldots \circ S_{e_{n}}$, and let

$$
J_{u}^{(n)}=\bigcup_{e \in E_{u}^{(n)}} S_{e}\left(J_{t(e)}\right) .
$$


Then

$$
K_{u}=\bigcap_{n=1}^{\infty} J_{u}^{(n)} .
$$

There is a model map $h_{u}: E_{u}^{(\omega)} \longrightarrow X_{u}$ for each $u \in V$, defined so that $h_{u}(\sigma)$ is the unique element of the set $\bigcap_{n=1}^{\infty} S_{\sigma \mid n}\left(J_{t(\sigma \mid n)}\right)$. So $K_{u}=h_{u}\left(E_{u}^{(\omega)}\right)$. For $\tau=$ $\tau_{1} \tau_{2} \ldots \tau_{n} \in E^{\star}$, we let $K(\tau)=S_{\tau}\left(K_{t(\tau)}\right), r(\tau)=r\left(\tau_{1}\right) r\left(\tau_{2}\right) \ldots r\left(\tau_{n}\right), p(\tau)=$ $p\left(\tau_{1}\right) p\left(\tau_{2}\right) \ldots p\left(\tau_{n}\right), r_{\max }=\max \{r(e): e \in E\}, r_{\min }=\min \{r(e): e \in E\}, p_{\max }=$ $\max \{p(e): e \in E\}, p_{\text {min }}=\min \{p(e): e \in E\}$. Let $G$ be a $M W$ graph. For $s \geq 0$, define a matrix $A_{s}$ with rows and columns indexed by $V$ :

$$
A_{s}=\left(\sum_{e \in E_{u v}} r_{e}^{s}\right)_{u, v} .
$$

Let $\Phi(s)$ be the spectral radius of $A_{s}$. The solution of $\Phi(s)=1$ is the dimension of the graph $G$ [14. For $\gamma \in E_{u v}^{\star}$, we have $p(\gamma)=\sum_{e \in E_{u}} p(\gamma e)$. Therefore, for each $u \in V$, there is a unique measure $\hat{\mu}_{u}$ on $E_{u}^{(\omega)}$ with $\hat{\mu}_{u}([\gamma])=p(\gamma), \quad \forall \gamma \in E_{u}^{\star}$. Let $\mu_{u}=\hat{\mu}_{u} \circ h_{u}^{-1}$. Then Hutchinson [13] has shown that $\mu_{u}$ is the unique probability measure supported by $K_{u}$, such that

$$
\mu_{u}=\sum_{e \in E_{u}} p_{e} \mu_{t(e)} \circ S_{e}^{-1} .
$$

The measure $\mu_{u}$ is called the self similar measure corresponding to the $M W$ graph. For $\tau \in E_{u}^{\star}$, let $J_{u}(\tau)=S_{\tau}\left(J_{t(\tau)}\right)$. Let $A(q, \beta)$ be the square matrix with rows and columns indexed by $V$ as follows:

$$
A_{u v}(q, \beta)=\sum_{e \in E_{u v}} p(e)^{q} r(e)^{\beta} .
$$

For a given $q$, there exists a unique $\beta$ so that the spectral radius of $A(q, \beta)$ is 1 . Since $A(q, \beta)$ has spectral radius 1 , there exist positive right and left eigenvectors $\rho_{v}, \lambda_{v}$ with

$$
\begin{aligned}
& \sum_{v \in V} \sum_{e \in E_{u v}} p(e)^{q} r(e)^{\beta} \rho_{v}=\rho_{u}, \quad \forall u \in V, \\
& \sum_{u \in V} \sum_{e \in E_{u v}} \lambda_{u} p(e)^{q} r(e)^{\beta}=\lambda_{v}, \quad \forall v \in V .
\end{aligned}
$$

By the Perron-Frobenius Theorem [20], $\lambda_{v}, \rho_{v}>0$. Let

$$
P(e)=\rho_{u}^{-1} p(e)^{q} r(e)^{\beta} \rho_{v} .
$$

Consider the Markov chain $\left(X_{k}\right)$ with state space $V$, and transition probabilities $P(e)$. Then $\pi_{u}=\lambda_{u} \rho_{u}, u \in V$, constitute a stationary distribution for the Markov chain.

The transition probabilities $P(e)$ also give rise to the measure $\hat{\mu}_{u}^{(q)}, q \in \mathbb{R}$, where each cylinder $[\gamma], \gamma \in E_{u v}^{(k)}$, is assigned the measure

$$
\hat{\mu}_{u}^{(q)}([\gamma])=\rho_{u}^{-1} p(\gamma)^{q} r(\gamma)^{\beta} \rho_{v} .
$$

These measures were defined and studied by Edgar and Mauldin [10]. For our purposes, we need a measure defined for the entire graph. This may be done using 
the stationary distribution. Let

$$
P^{\prime}(e)=\pi_{u} P(e), \quad \text { where } e \in E_{u v} .
$$

For $\gamma \in E^{\star}$, we let

$$
\hat{\mu}^{(q)}([\gamma])=\lambda_{i(\gamma)} p(\gamma)^{q} r(\gamma)^{\beta} \rho_{t(\gamma)} .
$$

This gives us a unique probability measure $\hat{\mu}^{(q)}$ on $E^{(\omega)}$. It is therefore clear from the uniqueness of the extended measures that

$$
\hat{\mu}_{u}^{(q)}=\frac{\left.\hat{\mu}^{(q)}\right|_{E_{u}^{(\omega)}}}{\hat{\mu}^{(q)}\left(E_{u}^{(\omega)}\right)} .
$$

Consider once again the matrix $A(q, \beta)$, whose entry in row $u$ and column $v$ is

$$
A_{u v}(q, \beta)=\sum_{e \in E_{u v}} p(e)^{q} r(e)^{\beta} .
$$

For a given $q$, let $\Phi(q, \beta)$ be the spectral radius of $A(q, \beta)$, and let $\beta$ be the unique value such that $\Phi(q, \beta)=1$. This defines $\beta$ as an analytic function of $q$. Define $\alpha=-\frac{d \beta}{d q}$ and $f(\alpha)=q \alpha+\beta$. We may sometimes write $\alpha=\alpha_{\mu}$ to emphasize its dependence on the measure $\mu$. These functions have been studied by [10].

For $x \in K_{u}, \alpha \in \mathbb{R}, \alpha>0$, we will call $\alpha$ the local dimension of $\mu$ at $x$ if

$$
\lim _{\epsilon \rightarrow 0} \frac{\log \mu\left(B_{\epsilon}(x)\right)}{\log \operatorname{diam}\left(B_{\epsilon}(x)\right)}=\alpha .
$$

Note that the existence of the limit is part of the definition. Most of our attention will be directed toward the following sets:

$$
\begin{aligned}
& K_{u}^{(\alpha)}=\left\{x \in K_{u}: \lim _{\epsilon \rightarrow 0} \frac{\log \mu_{u}\left(B_{\epsilon}(x)\right)}{\log \operatorname{diam}\left(B_{\epsilon}(x)\right)}=\alpha\right\}, \\
& \widehat{K}_{u}^{(\alpha)}=\left\{\sigma \in E_{u}^{(\omega)}: \lim _{k \rightarrow \infty} \frac{\log p(\sigma \mid k)}{\log r(\sigma \mid k)}=\alpha\right\} .
\end{aligned}
$$

Since we will be dealing with ratios of logarithms, we will adopt the following conventions. For $0<\varrho, \varsigma<1$,

$$
\begin{aligned}
& \frac{\log \varrho}{\log 0}=\frac{\log 1}{\log \varsigma}=\frac{\log 1}{\log 0}=0, \\
& \frac{\log 0}{\log \varsigma}=\frac{\log \varrho}{\log 1}=\frac{\log 0}{\log 1}=\infty, \\
& \frac{\log 0}{\log 0}=\frac{\log 1}{\log 1}=1 .
\end{aligned}
$$

The following result essentially allows us to move "pointwise" from the space of infinite strings to the Euclidean space. It was proven in [7] that:

Theorem 3.2. There exists a set $D_{u} \subseteq E^{(\omega)}$ such that for $x \in K_{u} \backslash h_{u}\left(D_{u}\right)$,

$$
x \in K_{u}^{(\alpha)} \Longleftrightarrow h_{u}^{-1}(x)=\sigma \in \widehat{K}_{u}^{(\alpha)} .
$$

Moreover, $\mu_{u}^{(q)}\left(h_{u} D_{u}\right)=0$ for every $q \in \mathbb{R}$. 
3.2. Dimension using Lebesgue measure. Consider the invariant set, $K_{u}$. Let $F \subseteq K_{u}$. Let $C_{u}$ be the collection of all the cylinder sets in the construction of $K_{u}$. Clearly, $C_{u}$ is a Vitali cover for $K_{u}$. Let $\operatorname{dim}^{c}$ denote the centered Hausdorff dimension. It is our purpose in this section to first show that

$$
\operatorname{dim} F=\operatorname{dim}^{c} F=\operatorname{dim}\left(F: C_{u}\right) .
$$

Then we will use Theorems 3.1 and 3.2 to obtain the Hausdorff dimensions of some multifractal sets.

We wish to begin with the following geometrical result, which is motivated by Schief [19, but first we need a definition:

Given $k \in E_{u}^{\star}, x \in K_{u} \cap J_{u}(k), \alpha>0$, let

$$
I(k, x, \alpha)=\left\{\tau \in E_{u}^{\star}: r(\tau) \leq r(k)<r\left(\tau^{-}\right) ; J_{u}(\tau) \cap B_{\alpha}(x) \neq \emptyset\right\} .
$$

A different form of the following proposition is given by Lemma 4.1.

Proposition 3.3. Let $G=(V, E)$ satisfy the $O S C$. Let $c>0$ be given. Then there exists a constant $\gamma$ such that:

For any $u \in V$, and $k \in E_{u}^{\star}$, any $x \in J_{u}(k)$, and any $\alpha>0$ with $\alpha \leq \operatorname{cr}(k)$, and $x \in J_{u}(k) \subseteq B_{\alpha}(x)$, we must have

$$
\# I(k, x, \alpha) \leq \gamma
$$

Proof. Let $u \in V$. Let $J_{u}$ be the compact set guaranteed by $O S C$. Let $c>0$ be given and let $y \in K_{u} \cap \operatorname{int}\left(J_{u}\right)$. Then there is an $\epsilon>0$ such that $B_{\epsilon}(y) \subseteq \operatorname{int}\left(J_{u}\right)$. For any $\tau \in E^{\star}$,

$$
S_{\tau}\left(B_{\epsilon}(y)\right)=B_{\epsilon r(\tau)}\left(S_{\tau}(y)\right) .
$$

Let $\sigma, \tau \in I(k, x, \alpha)$. Clearly, there is no $s \in E^{\star}$, such that $\tau=\sigma s$ or $\sigma=\tau s$ (i.e. $\sigma, \tau$ are incomparable). Thus,

$$
S_{\sigma}\left(\operatorname{int}\left(J_{u}\right)\right) \cap S_{\tau}\left(\operatorname{int}\left(J_{u}\right)\right)=\emptyset .
$$

Since, $B_{\epsilon}(y) \subseteq \operatorname{int}\left(J_{u}\right)$, we obtain

$$
\begin{aligned}
S_{\sigma}\left(B_{\epsilon}(y)\right) \cap S_{\tau}\left(B_{\epsilon}(y)\right) & =\emptyset \\
\Rightarrow B_{\epsilon r(\sigma)}\left(S_{\sigma}(y)\right) \cap B_{\epsilon r(\tau)}\left(S_{\tau}(y)\right) & =\emptyset \\
\Rightarrow d\left(S_{\sigma}(y), S_{\tau}(y)\right) & \geq(r(\sigma)+r(\tau)) \epsilon,
\end{aligned}
$$

where $d$ is the Euclidean metric in $\mathbb{R}^{d}$.

Since $\sigma, \tau \in I(k, x, \alpha)$, we obtain $r(\sigma) \leq r(k)<r\left(\sigma^{-}\right)$. Thus, $r(k)<\frac{r\left(\sigma^{-}\right)}{r_{\text {min }}}$.

Similarly, $r(\tau)>r_{\min } r(k)$. So we get,

$$
d\left(S_{\sigma}(y), S_{\tau}(y)\right) \geq 2 r_{\min } r(k) \epsilon .
$$

Consider the balls of radius $\frac{\epsilon}{2} r(k) r_{\min }$ around the points $S_{\tau}(y): \tau \in I(k, x, \alpha)$. These balls are pairwise disjoint, and their centres, $S_{\tau}(y)$, lie in a larger ball of radius $\left(\frac{\epsilon}{2} r(k) r_{\text {min }}+\operatorname{diam} B_{\alpha}(x)\right)$, centred at $x$. Using Lebesgue measure,

$$
\begin{aligned}
\# I(k, x, \alpha) & \leq\left(\frac{2 \frac{\epsilon}{2} r(k) r_{\min }+\operatorname{diam} B_{\alpha}(x)}{\epsilon r(k) r_{\min }}\right)^{d} \\
& \leq\left(\frac{\epsilon r_{\min }+2 c}{\epsilon r_{\min }}\right)^{d} .
\end{aligned}
$$


We now go on to the main result of this section.

Theorem 3.4. Let $u \in V$ and let $E \subseteq K_{u}$. Then

$$
\mathcal{H}^{\alpha}(E) \leq \mathcal{H}^{\alpha}\left(E: C_{u}\right) \leq \gamma 2^{\alpha} \mathcal{H}^{\alpha}(E) .
$$

Proof. It suffices to show that

$$
\mathcal{H}^{\alpha}\left(E: C_{u}\right) \leq \gamma 2^{\alpha} \mathcal{H}^{\alpha}(E) .
$$

Let $\delta>0$. Let $E \subseteq \bigcup_{j} B_{j}\left(x_{j}\right)$, where $x_{j} \in E$, $\operatorname{diam} B_{j}\left(x_{j}\right)<\delta$, and each $B_{j}\left(x_{j}\right)$ is a closed ball in $\mathbb{R}^{d}$. Since $C_{u}$ is a Vitali cover, there exists $I_{k(j)}\left(x_{j}\right) \in C_{u}$, such that

Then,

$$
\operatorname{diam}\left(I_{k(j)-1}\left(x_{j}\right)\right)>\operatorname{diam}\left(B_{j}\left(x_{j}\right)\right) \geq \operatorname{diam}\left(I_{k(j)}\left(x_{j}\right)\right) .
$$

$$
I_{k(j)}\left(x_{j}\right) \subseteq B_{j}\left(x_{j}\right)
$$

Also,

$$
\begin{aligned}
\operatorname{diam}\left(B_{j}\left(x_{j}\right)\right) & <\operatorname{diam}\left(I_{k(j)-1}\left(x_{j}\right)\right) \\
& =\frac{\operatorname{diam}\left(I_{k(j)}\left(x_{j}\right)\right)}{r_{k(j)}} \\
& <\frac{\operatorname{diam}\left(I_{k(j)}\left(x_{j}\right)\right)}{r_{\text {max }}} .
\end{aligned}
$$

By Proposition 3.3 we have

$$
E \cap B_{j}\left(x_{j}\right) \subseteq \bigcup_{k=1}^{\gamma} I_{k}(j)
$$

where

$$
I_{k}(j) \in I\left(k(j), x_{j}, \frac{\operatorname{diam}\left(B_{j}\left(x_{j}\right)\right)}{2}\right) .
$$

Moreover,

$$
\sum_{k=1}^{\gamma} \operatorname{diam}\left(I_{k}(j)\right)^{\alpha} \leq \gamma \operatorname{diam}\left(I_{k(j)}\left(x_{j}\right)\right)^{\alpha} \leq \gamma \operatorname{diam}\left(B_{j}\left(x_{j}\right)\right)^{\alpha},
$$

so that

$$
\begin{gathered}
\inf \left\{\sum_{j} \operatorname{diam}\left(I_{j}\right)^{\alpha}: E \subseteq \bigcup_{j} I_{j}, I_{j} \in g_{u}, \operatorname{diam}\left(I_{j}\right)<\delta\right\} \\
\leq \gamma \inf \left\{\sum_{j} \operatorname{diam}\left(B_{j}\left(x_{j}\right)\right)^{\alpha}: E \subseteq \bigcup_{j} B_{j}\left(x_{j}\right), x_{j} \in E, \operatorname{diam}\left(B_{j}\left(x_{j}\right)\right)<\delta\right\} .
\end{gathered}
$$

Letting $\delta \rightarrow 0$, we obtain

$$
\mathcal{H}^{\alpha}\left(E: C_{u}\right) \leq \gamma \widetilde{\mathcal{C}}^{\alpha}(E) \leq \gamma \mathcal{C}^{\alpha}(E) \leq \gamma 2^{\alpha} \mathcal{H}^{\alpha}(E),
$$

so

$$
\mathcal{H}^{\alpha}(E) \leq \mathcal{H}^{\alpha}\left(E: C_{u}\right) \leq \gamma 2^{\alpha} \mathcal{H}^{\alpha}(E)
$$

Corollary 3.5. Let $u \in V$ and let $E \subseteq K_{u}$. Then

$$
\operatorname{dim} E=\operatorname{dim}\left(E: C_{u}\right) .
$$


Theorem 3.6. Let $G=(V, E)$ be an $M W$ graph satisfying the $O S C, u \in V$ and let $\mu=\mu_{u}$ be any corresponding self-similar measure supported on $K_{u}$. If

$$
F \subseteq\left\{x \in K_{u}: \lim _{\epsilon \rightarrow 0} \frac{\log \mu\left(B_{\epsilon}(x)\right)}{\log \operatorname{diam}\left(B_{\epsilon}(x)\right)}=\theta\right\},
$$

then

$$
\operatorname{dim} F=\theta \operatorname{dim}_{\mu} F .
$$

In particular, if $\mu(F)>0$, then $\operatorname{dim} F=\theta$.

Proof. Let $x \in F$. By Theorem 3.2, we get

$$
\lim _{n \rightarrow \infty} \frac{\log p\left(c_{n}(x)\right.}{\log r\left(c_{n}(x)\right.}=\theta
$$

By Theorem 3.1 we obtain $\operatorname{dim}\left(F: C_{u}\right)=\theta \operatorname{dim}_{\mu} F$. By Corollary 3.5 we get $\operatorname{dim} F=\theta \operatorname{dim}_{\mu} F$.

As a corollary, we obtain a result proved in the random case in [1] and answer questions posed in [10 Question (d)] and [16, Question 7.8].

Corollary 3.7. $\operatorname{dim} K^{(\alpha)}=f(\alpha)$.

Proof. An easy computation (using Theorem 3.2) shows that

$$
x \in K^{(\alpha)} \Longleftrightarrow \lim _{\epsilon \rightarrow 0} \frac{\log \mu^{(q)}\left(B_{\epsilon}(x)\right)}{\log \operatorname{diam}\left(B_{\epsilon}(x)\right)}=f(\alpha) .
$$

Take $\mu=\mu^{(q)}$ in Theorem 3.6. Since $\mu^{(q)}\left(K^{(\alpha)}\right)=1$, the result follows.

Note that the proof of Theorem 3.4 depended heavily on the finiteness of the number of cylinder sets that may intersect a ball of a given radius, about the point $x_{j}$. This is essentially possible due to the uniform distribution and translation invariance of the Lebesgue measure. It therefore breaks down when we try to compute the Hausdorff dimension with respect to other measures. In an attempt to do this, we obtain a $b_{\mu}$-Vitali subcover which has this nice property.

\section{HAUSDORFF DIMENSION II}

Before we go any further with our discussions of the Hausdorff dimension, we need to create the appropriate framework. We will quote some pertinent definitions and results (without proofs) from [7]. In this and the next section, we show two applications of the notion of a $b_{\mu}$-Vitali subcover.

4.1. Preliminaries. The diameter of a cylinder set is nothing but the Lebesgue measure, suitably normalized. Thus all our notions and definitions make sense when considered with respect to measure $\nu$, where $\nu$ is defined by some different initial probability distribution. Suppose we assign two sets of probabilities $p(e), p^{\prime}(e)$ of traversing the edge $e$. Then we may change the matrix $A(q, \beta)$, so that its entry in row $u$ and column $v$ is

$$
A_{u v}(q, \beta)=\sum_{e \in E_{u v}} p(e)^{q} p^{\prime}(e)^{\beta}
$$


Let $\mu, \nu$ be the probability measures corresponding to the probabilities $p(e), p^{\prime}(e)$, $e \in E$, respectively. Then we may define the functions $\beta_{\mu, \nu}$ and $\alpha_{\mu, \nu}$ as before. Then standard computations show that $\alpha_{\mu, \nu}=\frac{\alpha_{\mu}}{\alpha_{\nu}}$, and similarly, we may obtain $f\left(\alpha_{\mu, \nu}\right)=\frac{f\left(\alpha_{\mu}\right)}{f\left(\alpha_{\nu}\right)}$. Also it is easy to deduce that $\Phi(0,1)=1=\Phi(1,0)$, where $\Phi(q, \beta)$ is the spectral radius of $A(q, \beta)$ defined in terms of $p(e), p^{\prime}(e), e \in E$.

For $K \subseteq \mathbb{R}^{d}, \alpha \in \mathbb{R}$, let

$$
K^{(\alpha)}=\left\{x \in \operatorname{supp} \mu: \lim _{\epsilon \rightarrow 0} \frac{\log \mu\left(B_{\epsilon}(x)\right)}{\log \nu\left(B_{\epsilon}(x)\right)}=\alpha\right\} .
$$

We are interested in the $\mu$-Hausdorff dimension of this set.

Let $E_{u}^{(1)}=\left\{e_{1}, e_{2}, \ldots, e_{u}\right\}$ and let $J_{u}\left(e_{k}\right)=S_{e_{k}}\left(J_{t\left(e_{k}\right)}\right), \quad k=1,2, \ldots, u$. Let $\tau \in E_{u}^{\star}$. Then $\tau$ is called a $u$-stopping iff

$$
K(\tau) \cap J_{u}\left(e_{k}\right)=\emptyset, \forall k=1, \ldots, u ; k \neq \tau \mid 1 .
$$

For each $u \in V, v \in N(u)$, there exists $e \tau_{v} \in E_{v}^{\star}$, such that $e \tau_{v}$ is a stopping for every $e \in E_{u v}$, and that moreover, $\left|e \tau_{v}\right|=m, \forall u \in V, v \in N(u), e \in E_{u v}$. Let

$$
\begin{gathered}
\bar{S}_{v}=\left\{s \in E_{v}^{\star}:|s|=\left|\tau_{v}\right|, e s \text { is a stopping for every } e \in E_{u v}\right\}, \\
S_{u}=\left\{e s: s \in \bar{S}_{v}, v \in N(u), e \in E_{u v}\right\} .
\end{gathered}
$$

Let $S=\bigcup_{u \in V} S_{u}$ denote the set of stoppings of length $m$, i.e. for every $\tau \in$ $S,|\tau|=m$. Let $\tau \in S_{u}$. Suppose $\tau=e \tau_{1} \tau_{2} \ldots \tau_{m-1}$, where $e \in E_{u v}$ for some $v \in N(u)$. Then $\tau^{-}=e \tau_{1}, \ldots, \tau_{m-2}$. Let $d(\tau)=\min \left\{d(x, y): x \in \partial J_{u}(\tau), y \in\right.$ $\left.\partial J_{u}\left(e_{k}\right), e_{k} \in E_{u}^{(1)}, e_{k} \neq e\right\}$. If $r\left(\tau^{-}\right)<d(\tau)$, let $N_{\tau}=1$. If $r\left(\tau_{-}\right) \geq d(\tau)$, then choose $N_{\tau} \in \mathbb{N}$, smallest, such that $r\left(\tau^{-}\right) r_{\max }^{N_{\tau}}<d(\tau)$. Clearly, such $N_{\tau}$ exists. Let $N_{r}=\max _{\tau \in S} N_{\tau}$.

For $\sigma \in E_{u}^{(\omega)}$, let $A_{i}$ be the event that $\sigma_{i} \sigma_{i+1} \ldots \sigma_{i+m-1} \in S, \quad X_{i}=1_{A_{i}}$, and choose $j_{k}(\sigma)$ such that

$$
\sum_{k=0}^{j_{k}(\sigma)} X_{i}=k+1
$$

If $x \in K_{u}$ such that $h_{u}^{-1}(x)=\sigma$ is unique, then let

$$
s_{k}(\sigma)=s_{k}(x)=j_{k}(\sigma)+N_{r}+m-1 .
$$

When $\sigma$ is fixed or its meaning is clear from the context, then we will write $j_{k}(\sigma)=$ $j_{k}, s_{k}(\sigma)=s_{k}$. Let

$$
\begin{aligned}
\widehat{K}^{(\alpha)} & =\bigcup_{u \in V} \widehat{K}_{u}^{(\alpha)}, \\
D_{u} & =\left\{\sigma \in E_{u}^{(\omega)}: \frac{k}{j_{k}(\sigma)} \rightarrow 0\right\}, \\
D & =\bigcup_{u \in V} D_{u}, \\
K & =\bigcup_{u \in V} K_{u} .
\end{aligned}
$$

Given $k \in E_{u}^{\star}, x \in K_{u} \cap J_{u}(k), \alpha>0$, let

$$
I_{\mu}(k, x, \alpha)=\left\{\tau \in E_{u}^{\star}: p(\tau) \leq p(k)<p\left(\tau^{-}\right) ; J_{u}(\tau) \cap B_{\alpha}(x) \neq \emptyset\right\} .
$$


Lemma 4.1 ([7], Lemma 3.5). Let $u \in V$ and let SOSC hold. Let $\sigma=\tau_{1} \tau \tau_{2}$, where $\tau_{1} \in E^{\star}$ with $t\left(\tau_{1}\right)=u, \tau \in S_{u}, \tau_{2} \in E_{t(\tau)}^{N_{r}}$. Then there is a constant $\gamma$ such that:

For any $\sigma \in E^{\star}$ as above, any $x \in J_{i\left(\tau_{1}\right)}(\sigma)$, any $u \in V$, and any $\alpha>0$ with

$\alpha \leq r\left(\sigma^{-}\right)$, and $x \in J_{i\left(\tau_{1}\right)}(\sigma) \subseteq B_{\alpha}(x)$, we must have

$$
\# I_{\mu}(\sigma, x, \alpha) \leq \gamma \text { and } \# I(\sigma, x, \alpha) \leq \gamma \text {. }
$$

It is known that for $q, \alpha$, as defined earlier, we have $\hat{\mu}^{(q)}\left(\widehat{K}^{(\alpha)}\right)=1$. Given $k \in \mathbb{N}$, let $\epsilon_{k}(x)=\epsilon_{k}(\sigma)=\max \left\{d(x, y): y \in \partial J_{u}(\sigma \mid k)\right\}$. We need the following result.

Lemma 4.2 ([7], Lemma 3.9). Let $x \in K_{u}$ such that $h_{u}^{-1}(x)=\sigma$ is unique. Then

(i) $x \in \bigcap_{k} J_{u}\left(\sigma \mid s_{k}\right)$.

(ii) $x \in J_{u}\left(\sigma \mid s_{k}\right) \subseteq B_{\epsilon_{s_{k}}}(x)$ and

$$
K_{u} \cap B_{\epsilon_{s_{k}}}(x) \subseteq \bigcup_{t \in I_{\mu}\left(\sigma \mid s_{k}, x, \epsilon_{s_{k}}\right)} J_{u}(t) \subseteq J_{u}\left(\sigma \mid j_{k}-1\right) \subseteq J_{u}\left(\sigma \mid j_{k-1}\right) .
$$

Let $S$ denote the set of stoppings, where we choose all our stoppings to have length $m$. For an infinite string $\sigma$, let $X_{k}(\sigma)=1$ if $\sigma_{k} \sigma_{k+1} \ldots \sigma_{k+m-1} \in S$ and 0 otherwise. Suppose $j_{k}(\sigma)$ denotes the $k+1$-st occurrence of a stopping in $\sigma$. Let $s_{k}=j_{k}(\sigma)+N_{r}+m-1$. For a given $\sigma$, let $S(\sigma)$ denote the set of all $e \in E$ such that $e$ occurs infinitely often in $\sigma$. Let $N_{k}(\sigma, \tau)$ denote the number occurrences of a fixed finite string $\tau$ in the first $k$ positions of $\sigma$. When $\sigma$ is fixed, we will simply say $N_{k}(\tau)$. We now make the following two assumptions:

(A1) $0<\lim _{k} \frac{N_{s_{k}}(e)}{s_{k}}<1 \forall e \in S(\sigma)$.

(A2) $\lim _{k} \frac{s_{k-1}}{s_{k}} \rightarrow 1$.

Lemma 4.3. Let $A$ denote the set of all infinite strings that satisfy (A1) and (A2). Then $\widehat{\mu}^{(q)}(A)=1$ for every $q \in \mathbb{R}$.

Proof. We will need to modify our stoppings. Let $S^{\prime}$ denote the modified stoppings, where each stopping is now of length $M$, and $M$ is chosen to ensure that if we look at all paths of length $M$ in $G$, then we obtain a new graph $G^{\prime}$ which is again strongly connected.

Let $\sigma$ be an infinite string that satisfies (A1) and $\lim _{k} \frac{N_{k M}(\tau)}{k M}>0$ for some $\tau \in S^{\prime}$. Then $\sigma$ will satisfy (A2) also. These two conditions, in turn, are certainly satisfied by any string $\sigma$ that has the following two properties:

(i) $0<\lim _{k} \frac{N_{k}(e)}{k}<1 \forall e \in E$.

(ii) $0<\lim _{k} \frac{N_{k}(\tau)}{k}<1 \forall \tau \in E^{(M)}$.

Now $G$ and $G^{\prime}$ are strongly connected and so by the ergodic theorem, these two properties are satisfied $\widehat{\mu}^{(q)}$-almost always. So for each $q \in \mathbb{R}$, we may choose strings that possess these properties, and these will then also satisfy assumptions (A1) and (A2).

Lemma 4.4. Let $\sigma \in E_{u}^{(\omega)}$ and $x=h_{u}(\sigma)$. Suppose $\sigma$ satisfies (A1) and (A2). Then

(i) $\lim _{k} \frac{\log p\left(\sigma \mid s_{k}\right)}{\log r\left(\sigma \mid s_{k}\right)}=\lim _{k} \frac{\log \mu\left(B_{\epsilon_{s_{k}}}(x)\right)}{\log \operatorname{diam}\left(B_{\epsilon_{s_{k}}}(x)\right)}$ and these limits exist. 
(ii) $\liminf _{k} \frac{\log p(\sigma \mid k)}{\log r(\sigma \mid k)}=\liminf _{\epsilon} \frac{\log \mu\left(B_{\epsilon}(x)\right)}{\log \operatorname{diam}\left(B_{\epsilon}(x)\right)}$ and this value is equal to the value of the limits in (i).

(iii) $\limsup _{k} \frac{\log p(\sigma \mid k)}{\log r(\sigma \mid k)}=\limsup _{\epsilon} \frac{\log \mu\left(B_{\epsilon}(x)\right)}{\log \operatorname{diam}\left(B_{\epsilon}(x)\right)}$.

Proof. The proof is the same as that for Propositions 4.3 and 4.4 in [7.

Lemma 4.5. Let $\sigma \in E_{u}^{(\omega)}$ and let $x=h_{u}(\sigma)$. Suppose $\sigma$ satisfies

(i) $0<\lim _{k} \frac{N_{k}(e)}{k}<1 \forall e \in E$.

(ii) $\lim _{k} \frac{N_{k}(\tau)}{k}>0$ for some $\tau \in S^{\prime}$. Then

$$
\lim _{k} \frac{\log p(\sigma \mid k)}{\log r(\sigma \mid k)}=\lim _{\epsilon} \frac{\log \mu\left(B_{\epsilon}(x)\right)}{\log \operatorname{diam}\left(B_{\epsilon}(x)\right)},
$$

and these limits exist.

Proof. Under the given assumtions, $\lim _{k} \frac{\log p(\sigma \mid k)}{\log r(\sigma \mid k)}$ exists. The result follows from Lemma 4.4

The following result ( 7 , Theorem 4.2]) essentially allows us to move "pointwise" from the space of infinite strings to the Euclidean space:

Theorem 4.6. Let $\sigma \in E_{u}^{(\omega)}, x=h_{u}(\sigma)$ and suppose $\sigma$ satisfies (A1) and (A2). Then

$$
\lim _{k} \frac{\log p(\sigma \mid k)}{\log r(\sigma \mid k)}=\lim _{\epsilon} \frac{\log \mu\left(B_{\epsilon}(x)\right)}{\log \operatorname{diam}\left(B_{\epsilon}(x)\right)},
$$

whenever one of these limits exist. Moreover,

(i) (A1) and (A2) are valid $\widehat{\mu}^{(q)}$-almost everywhere for each $q \in \mathbb{R}$.

(ii) The given limits exist and are equal $\widehat{\mu}^{(q)}$-almost everywhere for each $q \in \mathbb{R}$.

Proof. Follows immediately from Lemmas $4.3,4.4$ and 4.5

We are now ready to analyze these general multifractal sets.

4.2. $\mu$-Hausdorff dimension. Let $F_{u}=K_{u} \backslash D_{u}$. For each $x \in F_{u}$, let $s_{k}(x)$ be as defined, $\mu=\mu_{u}$ be any probability measure on $F_{u}$ constructed as before and finally, let

$$
\begin{aligned}
B_{\mu} & =\left(B_{\epsilon_{s_{k}}}(x): k \in \mathbb{N}, x \in F_{u}\right), \\
B & =\left(B(x, r): x \in F_{u}, r>0\right), \\
C_{\mu} & =\left(J_{u}\left(\sigma \mid s_{k}\right): k \in \mathbb{N}, \sigma \in h_{u}^{-1}\left(F_{u}\right)\right), \\
C & =\left(J_{u}(\sigma \mid k): k \in \mathbb{N}, \sigma \in h_{u}^{-1}\left(F_{u}\right)\right) .
\end{aligned}
$$

Proposition 4.7. For $E \subseteq F_{u}$,

(1) $\mathcal{H}_{\mu}^{\alpha}\left(E: B_{\mu}\right)=\mathcal{H}_{\mu}^{\alpha}(E: B)$.

(2) $\mathcal{H}_{\mu}^{\alpha}\left(E: C_{\mu}\right)=\mathcal{H}_{\mu}^{\alpha}(E: C)$.

Proof. Both follow from the observation that $B_{\mu}$ is a strong $b_{\mu}$-Vitali cover of $E$ with respect to $B$, and $C_{\mu}$ is a strong $b_{\mu}$-Vitali cover of $E$ with respect to $C$, with the choice of $b_{\mu}=1$. It is then an immediate consequence of Theorem 2.4. 
Corollary 4.8. For $E \subseteq F_{u}$,

(1) $\operatorname{dim}_{\mu}(E: B)=\operatorname{dim}_{\mu}\left(E: B_{\mu}\right)$.

(2) $\operatorname{dim}_{\mu}(E: C)=\operatorname{dim}_{\mu}\left(E: C_{\mu}\right)$.

Theorem 4.9. Let $E \subseteq F_{u}$. Then

$$
\mathcal{H}_{\mu}^{\alpha}(E: B) \leq \mathcal{H}_{\mu}^{\alpha}(E: C) \leq \gamma \mathcal{H}_{\mu}^{\alpha}(E: B) .
$$

Proof. By Proposition 4.7 it suffices to show that

$$
\mathcal{H}_{\mu}^{\alpha}\left(E: B_{\mu}\right) \leq \mathcal{H}_{\mu}^{\alpha}\left(E: C_{\mu}\right) \leq \gamma \mathcal{H}_{\mu}^{\alpha}\left(E: B_{\mu}\right) .
$$

We first show $\mathcal{H}_{\mu}^{\alpha}\left(E: C_{\mu}\right) \leq \gamma \mathcal{H}_{\mu}^{\alpha}\left(E: B_{\mu}\right)$. Let $\delta>0$ be given and let $\left(B_{j}\left(x_{j}\right)\right)_{j}$ be a $\mu-\delta-B_{\mu}$ cover of $E$. By our definition of $B_{j}\left(x_{j}\right)$, we can choose $I_{k(j)}\left(x_{j}\right) \in C_{\mu}$ such that

$$
\operatorname{diam} B_{j}\left(x_{j}\right)=2 \max \left\{d\left(x_{j}, y\right): y \in \partial I_{k(j)}\left(x_{j}\right)\right\} .
$$

Then we have

and by Lemma 4.2(ii),

$$
I_{k(j)}\left(x_{j}\right) \subseteq B_{j}\left(x_{j}\right)
$$

where

$$
B_{j}\left(x_{j}\right) \cap K_{u} \subseteq \bigcup_{k=1}^{\gamma} I_{k}(j),
$$

$$
I_{k}(j) \in I_{\mu}\left(k(j), x_{j}, \frac{\operatorname{diam}\left(B_{j}\left(x_{j}\right)\right)}{2}\right) .
$$

Thus, by Lemma 4.1 we may obtain

$$
\sum_{k=1}^{\gamma} \mu\left(I_{k}(j)\right)^{\alpha} \leq \gamma \mu\left(I_{k(j)}\left(x_{j}\right)\right)^{\alpha} \leq \gamma \mu\left(B_{j}\left(x_{j}\right)\right)^{\alpha} .
$$

But $\delta>0$ was arbitrary. So,

$$
\mathcal{H}_{\mu}^{\alpha}\left(E: C_{\mu}\right) \leq \gamma \mathcal{H}_{\mu}^{\alpha}\left(E: B_{\mu}\right) .
$$

Now the reverse inequality:

Let us denote $B_{\mu}=\left(B_{j}(x): j \in \mathbb{N}, x \in F_{u}\right)$. Let $N=N_{r}+m-1$. Consider

$$
E_{k, m, n}=\left\{x \in E: \mu\left(B_{m}(x)\right)>\frac{1}{n} \text { and } j \geq m \Rightarrow \frac{\log \mu\left(B_{j}(x)\right)}{\log \mu\left(B_{j+N}(x)\right)} \geq 1-\frac{1}{k}\right\} .
$$

Then

$$
E=\bigcup_{k} \bigcup_{m} E_{k, m, n}
$$

Fix $k, m, n \in \mathbb{N}$. Let $\rho \leq \frac{1}{n}$ and let $\left(I_{i}\left(x_{i}\right)\right)_{i}$ be a $\mu-\rho-C_{\mu}$ cover of $E_{k, m, n}$. For each $i$, let $B_{j(i)}\left(x_{i}\right) \in B_{\mu}$ such that

$$
\operatorname{diam} B_{j(i)}\left(x_{i}\right)=2 \max \left\{d\left(x_{i}, y\right): y \in \partial I_{i}\left(x_{i}\right)\right\} .
$$

Then, we have

and by Lemma4.2(ii),

$$
I_{i}\left(x_{i}\right) \subseteq B_{j(i)}\left(x_{i}\right),
$$

$$
K_{u} \cap B_{j(i)+N}\left(x_{i}\right) \subseteq I_{i}\left(x_{i}\right) .
$$

By our choice of $\rho$, we must have $j(i) \geq m$. Since $x_{i} \in E_{k, m, n}$, we get

$$
\frac{\log \mu\left(B_{j(i)}\left(x_{i}\right)\right)}{\log \mu\left(B_{j(i)+N}\left(x_{i}\right)\right)} \geq 1-\frac{1}{k} \text {. }
$$


Therefore,

$$
\mu\left(B_{j(i)}\left(x_{i}\right)\right)^{\alpha} \leq \mu\left(B_{j(i)+N}\left(x_{i}\right)\right)^{\alpha\left(1-\frac{1}{k}\right)} \leq \mu\left(I_{i}\left(x_{i}\right)\right)^{\alpha\left(1-\frac{1}{k}\right)},
$$

from which it follows that

$$
\mathcal{H}_{\mu}^{\alpha}\left(E_{k, m, n}: B_{\mu}\right) \leq \mathcal{H}_{\mu}^{\alpha\left(1-\frac{1}{k}\right)}\left(E_{k, m, n}: C_{\mu}\right)
$$

so that

$$
\mathcal{H}_{\mu}^{\alpha}\left(E: B_{\mu}\right) \leq \mathcal{H}_{\mu}^{\alpha}\left(E: C_{\mu}\right)
$$

Corollary 4.10. Let $E \subseteq F_{u}$. Then

$$
\operatorname{dim}_{\mu}(E: B)=\operatorname{dim}_{\mu}(E: C) .
$$

We are now ready to analyse the more general multifractal sets.

Theorem 4.11. Let $G=(V, E)$ be an $M W$ graph satisfying the $O S C, u \in V$ and let $\mu=\mu_{u}, \nu=\nu_{u}$ be any corresponding self similar measures supported on $K_{u}$. If

$$
F \subseteq\left\{x \in K_{u}: \lim _{\epsilon \rightarrow 0} \frac{\log \mu\left(B_{\epsilon}(x)\right)}{\log \nu\left(B_{\epsilon}(x)\right)}=\theta\right\}
$$

then

$$
\operatorname{dim}_{\nu} F=\theta \operatorname{dim}_{\mu} F
$$

In particular, if $\mu(F)>0$, then $\operatorname{dim}_{\nu} F=\theta$.

Proof. Let $x \in F$. By Theorem 4.6, we get

$$
\lim _{n \rightarrow \infty} \frac{\log \mu\left(c_{n}(x)\right)}{\log \nu\left(c_{n}(x)\right)}=\theta .
$$

Using Theorem 3.1] we obtain $\operatorname{dim}_{\nu}(F: C)=\theta \operatorname{dim}_{\mu}(F: C)$. So by Theorem 4.9] we get $\operatorname{dim}_{\nu}(F: B)=\theta \operatorname{dim}_{\mu}(F: B)$. But $B$ is just a centered ball covering. Thus by definition, we get

$$
\operatorname{dim}_{\nu} F=\theta \operatorname{dim}_{\mu} F
$$

Corollary 4.12. Let $\alpha=\alpha_{\mu_{u}, \nu_{u}}$. Then $\operatorname{dim} K^{(\alpha)}=f(\alpha)$.

Proof. An easy computation (using Theorem 4.6) shows that

$$
x \in K^{(\alpha)} \Longleftrightarrow \lim _{\epsilon \rightarrow 0} \frac{\log \mu^{(q)}\left(B_{\epsilon}(x)\right)}{\log \nu^{(q)}\left(B_{\epsilon}(x)\right)}=f(\alpha) .
$$

Take $\mu=\mu^{(q)}, \nu=\nu^{(q)}$ in Theorem 4.11 Since $\mu^{(q)}\left(K^{(\alpha)}\right)=1$, the result follows. 


\section{Multifractal Hausdorff measures}

The multifractal Hausdorff measures were introduced by Olsen [16]. Let $q, p \in \mathbb{R}$ and let $\alpha(p), \alpha(q)$ be as defined in Section 3.1. Olsen showed that for a graphdirected self-similar measure $\mu$ with totally disconnected support, if $\alpha(p) \neq \alpha(q)$, then the multifractal Hausdorff measures $\mathcal{H}_{\mu_{u}}^{p, \beta(p)}$ and $\mathcal{H}_{\mu_{u}}^{q, \beta(q)}$ are mutually singular. It is open as to whether this remains true when the support of $\mu$ satisfies the SOSC.

In this section, we first generalize Olsen's definition in a natural way to take into account two different measures $\mu$ and $\nu$, and then use the notion of a strong $b_{\mu}$-Vitali cover and the techniques in Section 4.2 to show that these more general multifractal Hausdorff measures are also mutually singular, which in particular, provides an answer to [16, Question 7.9].

5.1. Preliminaries. For $E \subseteq \mathbb{R}^{d}$, let $\mathbb{B}=\left(B\left(x_{i}, r_{i}\right)\right)_{i}$ be the collection of all closed balls in $\mathbb{R}^{d}$. Let $\mathcal{P}\left(\mathbb{R}^{d}\right)$ denote the family of all Borel probability measures on $\mathbb{R}^{d}$. Let $\mu, \nu \in \mathcal{P}\left(\mathbb{R}^{d}\right)$. For $\delta>0, \mathbb{B}$ is called a centered $\nu-\delta$-covering of $E$ if $E \subseteq \bigcup_{i} B\left(x_{i}, r_{i}\right), x_{i} \in E$ and $0<\nu\left(B\left(x_{i}, r_{i}\right)\right)<\delta, \forall i$.

Let $\mathbb{B}$ be a centered $\nu-\delta$-covering of $E$. For $q, t \in \mathbb{R}$ we define the multifractal Hausdorff measure (with respect to $\nu$ ) as follows:

$$
\begin{aligned}
& \overline{\mathcal{H}}_{\mu, \nu, \delta}^{q, t}(E)= \inf \left\{\sum_{i} \mu\left(B\left(x_{i}, r_{i}\right)\right)^{q} \nu\left(B\left(x_{i}, r_{i}\right)\right)^{t}:\right. \\
&\left.E \subseteq \bigcup_{i} B\left(x_{i}, r_{i}\right), B\left(x_{i}, r_{i}\right) \in \mathbb{B} \forall i\right\}, \\
& \overline{\mathcal{H}}_{\mu, \nu, \delta}^{q, t}(\emptyset)=0, \\
& \overline{\mathcal{H}}_{\mu, \nu}^{q, t}(E)=\sup _{\delta>0} \overline{\mathcal{H}}_{\mu, \nu, \delta}^{q, t}(E), \\
& \mathcal{H}_{\mu, \nu}^{q, t}(E)=\sup _{F \subseteq E} \overline{\mathcal{H}}_{\mu, \nu}^{q, t}(F) .
\end{aligned}
$$

Let $V$ denote any of the collections $B, B_{\mu}, C, C_{\mu}$ as defined in Section 4.2. We will denote the corresponding multifractal Hausdorff measure by

$$
\mathcal{H}_{\mu, \nu}^{q, t}(E: V) .
$$

When we restrict our attention to subsets of a compact set $K$, Olsen's measures may be obtained by taking $\nu$ to be the Lebesgue measure suitably normalized to $K$, and thus may be replaced either by the diameter of the ball, $2 r_{i}$, or by the size of a cylinder set, $r(\gamma)$. Our main result may then be stated as follows:

Let $G=(V, E)$. Let $\mu, \nu$ be any two graph-directed self-similar measures satisfying $O S C$. If $q, p \in \mathbb{R}$ and $\alpha(p) \neq \alpha(q)$, then

$$
\mathcal{H}_{\mu, \nu}^{p, \beta(p)} \perp \mathcal{H}_{\mu, \nu}^{q, \beta(q)} .
$$

We briefly outline the proof: we may compare $\mathcal{H}_{\mu, \nu}^{q, \beta(q)}(E: C)$ and $\hat{\mu}^{(q)}$. We show that

$$
\begin{aligned}
\mathcal{H}_{\mu, \nu}^{q, t}\left(E: B_{\mu}\right) & =\mathcal{H}_{\mu, \nu}^{q, t}(E: B), \\
\mathcal{H}_{\mu, \nu}^{q, t}\left(E: C_{\mu}\right) & =\mathcal{H}_{\mu, \nu}^{q, t}(E: C) .
\end{aligned}
$$

Next, we show that there exist positive constants $C_{1}, C_{2}$, such that for all $q, t \in \mathbb{R}$,

$$
C_{1} \mathcal{H}_{\mu, \nu}^{q, t}(E: B) \leq \mathcal{H}_{\mu, \nu}^{q, t}(E: C) \leq C_{2} \mathcal{H}_{\mu, \nu}^{q, t}(E: B)
$$


Finally, we may use the mutual singularity of the measures $\mu^{(q)}$ and $\mu^{(p)}$ to deduce our result.

\subsection{Mutual singularity.}

Proposition 5.1. Let $F \subseteq \mathbb{R}^{d}$, let $\mu, \nu \in \mathcal{P}\left(\mathbb{R}^{d}\right)$, and let $V$ be a Vitali cover for $F$. If $V_{\mu}$ is a subcover such that it is a strong $b_{\mu}$-Vitali cover of $F$ with respect to $V$, and a strong $b_{\nu}$-Vitali cover of $F$ with respect to $V$, then for any $E \subseteq F, q, t \in \mathbb{R}$,

$$
\mathcal{H}_{\mu, \nu}^{q, t}\left(E: V_{\mu}\right)=\mathcal{H}_{\mu, \nu}^{q b_{\mu}, t b_{\nu}}(E: V) .
$$

Proof. Consider the set

$$
\begin{aligned}
E_{k, m, n}=\{x \in E & : \quad \nu\left(I_{m}(x)\right)>\frac{1}{n} \text { and } j \geq m \\
& \left.\Rightarrow \quad b_{p}-\frac{1}{k} \leq \frac{\log p\left(I_{j}(x)\right)}{\log p\left(I_{j+1}(x)\right)} \leq b_{p}+\frac{1}{k}, p=\mu, \nu\right\} .
\end{aligned}
$$

Then,

$$
E=\bigcup_{k} \bigcup_{m} E_{k, m, n}
$$

Fix $k, m, n \in \mathbb{N}$. Let $\rho \leq \frac{1}{n}$ and let $\left(B_{i}\right)$ be a $\nu-\rho$ cover of $E_{k, m, n}$ using sets from $V$. By the definition of $V_{\mu}$, we can choose $j(i)$ such that

$$
I_{j(i)+1}\left(x_{i}\right) \subseteq B_{i} \subseteq I_{j(i)}\left(x_{i}\right) .
$$

By our choice of $\rho, j(i) \geq m$. Since $x_{i} \in E_{k, m, n}$, we get

$$
p\left(I_{j(i)+1}\left(x_{i}\right)\right)^{\left(b_{p}+\frac{1}{k}\right)} \leq p\left(I_{j(i)}\left(x_{i}\right)\right) \leq p\left(I_{j(i)+1}\left(x_{i}\right)\right)^{\left(b_{p}-\frac{1}{k}\right)}, p=\mu, \nu .
$$

We show how the proof works for $q>0, t>0$ :

$$
\begin{aligned}
\sum \mu\left(I_{j(i)}\left(x_{i}\right)\right)^{q} \nu\left(I_{j(i)}\left(x_{i}\right)\right)^{t} & \leq \sum \mu\left(I_{j(i)+1}\left(x_{i}\right)\right)^{q\left(b_{\mu}-\frac{1}{k}\right)} \nu\left(I_{j(i)+1}\left(x_{i}\right)\right)^{t\left(b_{\nu}-\frac{1}{k}\right)} \\
& \leq \sum \mu\left(B_{i}\right)^{q\left(b_{\mu}-\frac{1}{k}\right)} \nu\left(B_{i}\right)^{t\left(b_{\nu}-\frac{1}{k}\right)},
\end{aligned}
$$

from which it follows that

$$
\mathcal{H}_{\mu, \nu}^{q, t}\left(E: V_{\mu}\right) \leq \mathcal{H}_{\mu, \nu}^{q b_{\mu}, t b_{\nu}}(E: V) .
$$

Similarly, by using the opposite estimate for the measures in Equation (5.1), we may obtain the reverse inequality. The other cases for $q, t \in \mathbb{R}$, may be dealt with by choosing the right combination of estimates in Equation (5.1).

From now on, we will be concerned with the graph directed measures. We will therefore write $\mu \equiv \mu_{u}$ and $\nu \equiv \nu_{u}$.

Corollary 5.2. For $E \subseteq F_{u}$,

(1) $\mathcal{H}_{\mu, \nu}^{q, t}\left(E: B_{\mu}\right)=\mathcal{H}_{\mu, \nu}^{q, t}(E: B)$.

(2) $\mathcal{H}_{\mu, \nu}^{q, t}\left(E: C_{\mu}\right)=\mathcal{H}_{\mu, \nu}^{q, t}(E: C)$.

Proof. Both follow from the observation that $B_{\mu}, C_{\mu}$ are strong $b_{\mu}$-Vitali and strong $b_{\nu}$-Vitali covers of $E$ with respect to $B, C$ respectively, and that $b_{\mu}=b_{\nu}=1$. It is then an immediate consequence of Proposition 5.1]

Proposition 5.3. Let $E \subseteq F_{u}$. Then there exist positive constants $C_{1}, C_{2}$, such that for all $q, t \in \mathbb{R}$,

$$
C_{1} \mathcal{H}_{\mu, \nu}^{q, t}(E: B) \leq \mathcal{H}_{\mu, \nu}^{q, t}(E: C) \leq C_{2} \mathcal{H}_{\mu, \nu}^{q, t}(E: B) .
$$


Proof. By Corollary 5.2, it suffices to show that

$$
C_{1} \mathcal{H}_{\mu, \nu}^{q, t}\left(E: B_{\mu}\right) \leq \mathcal{H}_{\mu, \nu}^{q, t}\left(E: C_{\mu}\right) \leq C_{2} \mathcal{H}_{\mu, \nu}^{q, t}\left(E: B_{\mu}\right)
$$

Let $\delta>0$ be given and let $\left(B_{j}\left(x_{j}\right)\right)_{j}$ be a $\nu-\delta-B_{\mu}$ cover of $E$. By our definition of $B_{j}\left(x_{j}\right)$, we can choose $I_{k(j)}\left(x_{j}\right) \in C_{\mu}$ such that

$$
\operatorname{diam} B_{j}\left(x_{j}\right)=2 \max \left\{d\left(x_{j}, y\right): y \in \partial I_{k(j)}\left(x_{j}\right)\right\} .
$$

Then we have

$$
I_{k(j)}\left(x_{j}\right) \subseteq B_{j}\left(x_{j}\right) .
$$

Using Lemma 4.1 with measure $\mu$ and $\nu$, along with Lemma4.2(ii), we may deduce that there are at most $2 \gamma$ cylinders such that

$$
B_{j}\left(x_{j}\right) \cap K_{u} \subseteq \bigcup_{k=1}^{2 \gamma} I_{k}(j)
$$

where

$$
I_{k}(j) \in I_{\mu}\left(k(j), x_{j}, \frac{\operatorname{diam}\left(B_{j}\left(x_{j}\right)\right)}{2}\right) \bigcup I_{\nu}\left(k(j), x_{j}, \frac{\operatorname{diam}\left(B_{j}\left(x_{j}\right)\right)}{2}\right) .
$$

Moreover,

$$
p\left(I_{k}(j)\right) \leq p\left(I_{k(j)}\left(x_{j}\right)\right) \leq p\left(B_{j}\left(x_{j}\right)\right), p=\mu, \nu .
$$

Thus for $q>0, t>0$, we obtain the following:

$$
\begin{aligned}
\sum_{k=1}^{2 \gamma} \mu\left(I_{k}(j)\right)^{q} \nu\left(I_{k}(j)\right)^{t} & \leq 2 \gamma \mu\left(I_{k(j)}\left(x_{j}\right)\right)^{q} \nu\left(I_{k(j)}\left(x_{j}\right)\right)^{t} \\
& \leq 2 \gamma \mu\left(B_{j}\left(x_{j}\right)\right)^{q} \nu\left(B_{j}\left(x_{j}\right)\right)^{t} .
\end{aligned}
$$

But $\delta>0$ was arbitrary. So,

$$
\mathcal{H}_{\mu, \nu}^{q, t}\left(E: C_{\mu}\right) \leq 2 \gamma \mathcal{H}_{\mu, \nu}^{q, t}\left(E: B_{\mu}\right) .
$$

Let us denote $B_{\mu}=\left(B_{j}\left(x_{j}\right): j \in \mathbb{N}, x \in F_{u}\right)$. Let $N=N_{r}+m-1$. Consider

$$
\begin{aligned}
E_{k, m, n}=\{x \in E \quad & \quad \nu\left(B_{m}(x)\right)>\frac{1}{n} \text { and } j \geq m \\
\Rightarrow & \left.1-\frac{1}{k} \leq \frac{\log p\left(B_{j}(x)\right)}{\log p\left(B_{j+N}(x)\right)} \leq 1+\frac{1}{k}, p=\mu, \nu\right\} .
\end{aligned}
$$

Then

$$
E=\bigcup_{k} \bigcup_{n} E_{k, m, n}
$$

Fix $k, m, n \in \mathbb{N}$; let $\rho \leq \frac{1}{n}$ and let $\left(I_{i}\left(x_{i}\right)\right)_{i}$ be a $\nu-\rho-C_{\mu}$ cover of $E_{k, m, n}$. For each $i$, let $B_{j(i)}\left(x_{i}\right) \in B_{\mu}$ such that

$$
\operatorname{diam} B_{j(i)}\left(x_{i}\right)=2 \max \left\{d\left(x_{i}, y\right): y \in \partial I_{i}\left(x_{i}\right)\right\} .
$$

Then, we have

$$
I_{i}\left(x_{i}\right) \subseteq B_{j(i)}\left(x_{i}\right)
$$

and by Lemma4.2(ii),

$$
K_{u} \cap B_{j(i)+N}\left(x_{i}\right) \subseteq I_{i}\left(x_{i}\right) .
$$


By our choice of $\rho$, we must have $j(i) \geq m$. Since $x_{i} \in E_{k, m, n}$, we get

$$
\begin{aligned}
\mu\left(B_{j(i)}\left(x_{i}\right)\right)^{q} \nu\left(B_{j(i)}\left(x_{i}\right)\right)^{t} & \leq \mu\left(B_{j(i)+N}\left(x_{i}\right)\right)^{q\left(1-\frac{1}{k}\right)} \nu\left(B_{j(i)+N}\left(x_{i}\right)\right)^{t\left(1-\frac{1}{k}\right)} \\
& \leq \mu\left(I_{i}\left(x_{i}\right)\right)^{q\left(1-\frac{1}{k}\right)} \nu\left(I_{i}\left(x_{i}\right)\right)^{t\left(1-\frac{1}{k}\right)}
\end{aligned}
$$

from which it follows that

$$
\mathcal{H}_{\mu, \nu}^{q, t}\left(E: B_{\mu}\right) \leq \mathcal{H}_{\mu, \nu}^{q, t}\left(E: C_{\mu}\right) .
$$

For the other choices of $q, t \in \mathbb{R}$, we may choose the appropriate estimate from Equation (5.2) and Equation (5.3).

Theorem 5.4. If $q, p \in \mathbb{R}, \alpha(q) \neq \alpha(p)$, then

$$
\mathcal{H}_{\mu, \nu}^{p, \beta(p)} \perp \mathcal{H}_{\mu, \nu}^{q, \beta(q)} .
$$

Proof. Observe that for any $E \subseteq F_{u}$,

$$
\left(\rho_{\min } / \rho_{\max }\right) \mathcal{H}_{\mu, \nu}^{q, \beta(q)}(E: C) \leq \hat{\mu}^{(q)}\left(h_{u}^{-1} E\right) \leq\left(\rho_{\max } / \rho_{\min }\right) \mathcal{H}_{\mu, \nu}^{q, \beta(q)}(E: C) .
$$

By Proposition 5.3 we obtain

$$
\left(\rho_{\min } / \rho_{\max }\right) C_{1} \mathcal{H}_{\mu, \nu}^{q, \beta(q)}(E: B) \leq \mu^{(q)}(E) \leq\left(\rho_{\max } / \rho_{\min }\right) C_{2} \mathcal{H}_{\mu, \nu}^{q, \beta(q)}(E: B) .
$$

But for $q, p \in \mathbb{R}, \alpha(q) \neq \alpha(p)$,

$$
\mu^{(q)} \perp \mu^{(p)} .
$$

Hence the result follows.

Much of this work was done as part of my doctoral dissertation [5].

\section{REFERENCES}

1. Matthias Arbeiter and Norbert Patzschke, Random Self Similar Multifractals, Math. Nachr. 181 (1996), 5-42. MR 1409071 (97j:28016)

2. P. Billingsley, Hausdorff Dimension in Probability Theory II, Ill. J. Math. 5 (1961), 291-298. MR0120339 (22:11094)

3. R. Cawley and R. D. Mauldin, Multifractal Decomposition of Moran Fractals, Adv. in Math. 92 (1992), 196-236. MR1155465 (93b:58085)

4. C. D. Cutler, A note on equivalent interval covering systems for Hausdorff dimension on $\mathbf{R}$, Int. J. Math. Math. Sci. 11 (1988), no. 4, 643-650. MR0959443 (89h:28008)

5. Manabendra Das, Pointwise Local Dimensions, Ph.D. Thesis, The Ohio State University, 1996.

6. Manav Das, Binary Expansions and Multifractals, Fractal Frontiers, World Scientific Publishers, (1997), 131-139. MR1636266 (99g:28008)

7. Manav Das, Local Properties of Self-Similar Measures, Ill. J. Math. 42 (1998), no. 2, 313-332. MR 1612763 (99c:28012)

8. Manav Das, Packings and Pseudo - Packings: Measures, Dimensions and Mutual Singularity, preprint.

9. G. A. Edgar, Measure, topology, and fractal geometry, Springer-Verlag, New York, 1990. MR1065392 (92a:54001)

10. G. A. Edgar and R. D. Mauldin, Multifractal decomposition of digraph recursive fractals, Proc. Lond. Math. Soc. 65 (1992), 604-628. MR1182103 (93h:28010)

11. K. J. Falconer, Fractal geometry. Mathematical foundations and applications, John Wiley \& Sons, Ltd., Chichester, 1990. MR1102677 (92j:28008)

12. T. C. Halsey, M. H. Jensen, L. P. Kadanoff, I. Procaccia and B. J. Shraiman, Fractal Measures and their singularities: The characterization of strange sets, Phys. Rev. A 33 (1986), 11411151. MR 0823474 (87h:58125a)

13. J. E. Hutchinson, Fractals and self-similarity, Indiana Univ. Math. J. 30 (1981), 713-747. MR0625600 (82h:49026) 
14. R. D. Mauldin and S. C. Williams, Hausdorff dimension in graph directed constructions, Trans. Amer. Math. Soc. 309 (1988), 811-829. MR0961615 (89i:28003)

15. Lars Olsen, Random Geometrically Graph Directed Self-Similar Multifractals, Pitman Research Notes in Mathematics Series, Vol. 307, Longman Scientific and Technical, 1994. MR 1297123 (95j:28006)

16. L. Olsen, A multifractal formalism, Adv. in Math. 116 (1995), no. 1, 82-196. MR1361481 (97a:28006)

17. Yakov B. Pesin, Dimension Theory in Dynamical Systems: Contemporary Views and Applications, The University of Chicago Press, 1997. MR.1489237 (99b:58003)

18. C. A. Rogers, Hausdorff Measures, Cambridge University Press, 1970. MR0281862 (43:7576)

19. A. Schief, Separation properties for self-similar sets, Proc. Amer. Math. Soc. 122 (1992), 111-115. MF $1191872(94 \mathrm{k}: 28012)$

20. E. Seneta, Non-negative Matrices, Wiley, 1973. MR0389944 (52:10773)

21. JingLing Wang, The open set conditions for graph directed self-similar sets, Random Comput. Dynam. 5 (1997), no. 4, 283-305. MR1483871 (99g:28019)|

Department of Mathematics, The Ohio State University, Columbus, Ohio 43210

Current address: Department of Mathematics, University of Louisville, Louisville, Kentucky 40292

E-mail address: manav@louisville.edu 